\title{
SNIPE score can capture prodromal Alzheimer's in cognitively normal subjects
}

\author{
Azar Zandifar ${ }^{1,2}$ (MSc) \\ azar.zandifar@mail.mcgill.ca \\ Vladimir Fonov ${ }^{1}(\mathrm{PhD})$ \\ vladimir.fonov@mcgill.ca \\ Olivier Potvin ${ }^{3}(\mathrm{PhD})$ \\ olivier.potvin@cervo.ulaval.ca \\ Simon Duchesne ${ }^{3,4}(\mathrm{PhD}) \quad \underline{\text { simon.duchesne@fmed.ulaval.ca }}$ \\ D. Louis Collins ${ }^{1,2}(\mathrm{PhD}) \quad \underline{\text { louis.collins@mcgill.ca }}$ \\ for the Alzheimer's Disease Neuroimaging Initiative ${ }^{1}$
}

1. McConnell Brain Imaging Centre, Montreal Neurological Institute, McGill University, Montreal, Canada

2. Department of Biomedical Engineering, McGill University, Montreal, Canada

3. CERVO Research Center, Quebec Mental Health Institute, Québec, Canada

4. Radiology Department, Université Laval, Québec, Canada

\section{Corresponding Author Information:}

Azar Zandifar,

McConnell Brain Imaging Centre, Montreal Neurological Institute

Montreal Neurological Institute, 3801 University Street, Room WB320, Montreal, QC, H3A 2B4

Email: azar.zandifar@mcgill.ca

Tel: +1-514-398-1573

1 Data used in preparation of this article were obtained from the Alzheimer's Disease Neuroimaging Initiative (ADNI) database (adni.loni.usc.edu). As such, the investigators within the ADNI contributed to the design and implementation of ADNI and/or provided data but did not participate in analysis or writing of this report. A complete listing of ADNI investigators can be found at:

http://adni.loni.usc.edu/wp-ontent/uploads/how_to_apply/ADNI_Acknowledgement_List.pdf 


\title{
SNIPE score can capture prodromal Alzheimer's in cognitively normal subjects
}

\author{
Azar Zandifar, Valdimir S. Fonov, O. Potvin, S. Duchesne, D. Louis Collins
}

\begin{abstract}
Capturing early changes in the brain related to Alzheimer's disease may lead to models that successfully predict cognitive decline and the eventual onset of dementia, well ahead of onset of clinical symptoms. In this study we used both hippocampal volume and our hippocampal driven SNIPE score to show which marker better captures Alzheimer's related changes in a large dataset of normal controls $(\mathrm{N}=515)$ from the ADNI study, comparing controls that remain cognitively stable and controls that progress to either MCI or Alzheimer's dementia during 10 years of followup (median follow-up: 30 months). We measured hippocampal volume and SNIPE score and found that the effect size to differentiate between stable and progressor groups was significantly larger for SNIPE score than for volume. Our results also show that there is a significant age-related difference between groups for both markers, and the difference is greater with the SNIPE score. Our experiments show that considering high sensitivity of our SNIPE score regarding to early ADrelated brain changes, this marker is a better candidate in comparison to hippocampal volume for predicting the future onset of dementia.
\end{abstract}

\section{Introduction}

Alzheimer's disease (AD) is characterized by abnormal tau aggregation, concurrent to synaptic dysfunction, cell death, and brain atrophy (Oddo et al., 2006), as well as abnormal processing of the amyloid precursor protein, which leads to beta amyloid deposits in the cortex (Blennow, de 
Leon, \& Zetterberg, 2006). In its typical non-dominantly inherited form ( $>99 \%$ of cases)(Campion et al., 1999), sufficient evidence has been gathered from biomarker studies (C. R. Jack, Jr. et al., 2013) as well as post-mortem, pathological reports (Duyckaerts, 2011; Hyman et al., 2012) to postulate that these processes span more than two decades. Given such evidence, early detection of prodromal disease is key to intervene before the onset of too much irreversible neurodegeneration. This requires biomarkers specific to $\mathrm{AD}$ progression that are sufficiently sensitive decades ahead of diagnostic.

Radiological-pathological studies have confirmed that structural (T1-weighted) MRI tracks brain atrophy in AD (Csernansky et al., 2004; C. R. Jack, Jr. et al., 2002) at the global, lobar and regional level (Ashburner et al., 2003; Chetelat \& Baron, 2003; Csernansky et al., 2004; Fox \& Schott, 2004), shown to correspond to neuronal losses in layer II of the entorhinal cortex (Gomez-Isla et al., 1996), in hippocampal CA1 (West, Coleman, Flood, \& Troncoso, 1994), in the superior temporal gyrus (Gomez-Isla et al., 1997), and in the supramarginal gyrus (Grignon, Duyckaerts, Bennecib, \& Hauw, 1998). Hippocampal atrophy in particular has been thoroughly studied in AD (H. Braak \& Braak, 1991; Heiko Braak \& Braak, 1995), with a clear reported difference between patients and age-matched cognitively healthy $(\mathrm{CH})$ individuals (Coupé, Eskildsen, Manjón, Fonov, \& Collins, 2012; C. R. Jack et al., 1997; A. Zandifar et al., 2017). In those subjects with mild cognitive impairment (MCI), that is those with memory complaints who objectively show demonstrable cognitive abnormalities and biomarker evidence of AD pathology but do not meet criteria for dementia (Albert et al., 2011), hippocampal volume can predict progression on a single subject basis (Coupé, Eskildsen, Manjón, Fonov, \& Collins, 2012; A. Zandifar et al., 2017; Azar Zandifar, Fonov, Coupé, Pruessner, \& Collins, 2014). It has been shown as well that hippocampal volume shows very low percentage of abnormality in cognitively healthy cohorts, while abnormality grows with disease progression (C. R. Jack et al., 2013). Our recent study shows that 
classification of $\mathrm{AD}$ patients versus $\mathrm{CH}$ based on hippocampal volume yields an area under the receiver operating curve (AUC) for the AD Neuroimaging Initiative (ADNI) dataset of $88 \%$, while the AUC is $64 \%$ for differentiating MCI individuals that progressed to probable AD from those whose status remained stable for up to three years of follow-up, a result that did not depend on the different automatic segmentation methods tested (A. Zandifar et al., 2017). Hippocampal volume and atrophy stand therefore as putative biomarkers that could be used for early detection.

However, the Scoring by Nonlocal Image Patch Estimator (SNIPE) metric has been shown to surpass hippocampal volume in terms of predictive power, especially in the MCI stage (Coupé, Eskildsen, Manjón, Fonov, \& Collins, 2012; Coupe et al., 2015). When compared to hippocampal volume, the SNIPE score increased the accuracy of a single-subject prediction of dementia in the MCI population by 10 percent (Coupé, Eskildsen, Manjón, Fonov, Pruessner, et al., 2012). Inspired by nonlocal patch-based denoising (Coupe et al., 2008), SNIPE is a disease probability scoring method, estimated within the hippocampal and entorhinal cortices (Coupé, Eskildsen, Manjón, Fonov, \& Collins, 2012), which assigns a similarity score to each voxel that shows how much the patch around that specific voxel is similar to a library of probable AD patients or age-matched $\mathrm{CH}$ subjects.

Our aim in this study was to demonstrate the sensitivity of SNIPE scoring at detecting very early AD-related pathological changes in a cognitively healthy cohort. Our hypothesis was that there would be a difference in SNIPE scores between $\mathrm{CH}$ individuals that remained cognitively stable from those who declined, well before the onset of clinical symptoms; and that this difference was more emphasized using hippocampal SNIPE scoring than hippocampal volumetry. 


\section{Methods}

\section{Participants and clinical follow-up}

Data used in the preparation of this article were obtained from the Alzheimer's Disease Neuroimaging Initiative (ADNI) database (adni.loni.usc.edu). The ADNI was launched in 2003 as a public-private partnership, led by Principal Investigator Michael W. Weiner, MD. The primary goal of ADNI has been to test whether serial magnetic resonance imaging (MRI), positron emission tomography (PET), other biological markers, and clinical and neuropsychological assessment can be combined to measure the progression of mild cognitive impairment (MCI) and early Alzheimer's disease (AD). For up-to-date information, see www.adni-info.org.

The dataset used in this study is composed of the CH cohort of ADNI-1 and ADNI-2. They showed no signs of depression, mild cognitive impairment or dementia (Alzheimer's Disease Neuroimaging Initiative, 2018). The dataset consisted of $515 \mathrm{CH}$ individuals, 228 from ADNI-1 and the balance from ADNI-2. Each subject was followed throughout the study period (up to 10 years) to screen for any kind of future change in diagnostic label. We used the most recent clinical diagnostic information available for each subject to determine if these individuals remained stable or progressed to either MCI or AD. From our dataset of 515 normal controls at baseline, 427 maintained their cognitively normal status throughout the follow-up period and were thus labeled as Stables. The remaining 88 subjects converted to either MCI or AD and were labeled as Progressives. The median follow-up period (30 months) is the same for the two groups. Study phase, age, and sex were extracted from baseline reports, and are summarized in Table 1. 
Table 1. Dataset Information - The values in parentheses are $25 \%$ and $75 \%$ quantiles respectively.

\begin{tabular}{|l|l|l|}
\hline & Progressive & Stable \\
\hline Number & 88 & 427 \\
\hline Median Age at Baseline & $76.4(72.5-80.0)$ & $73.8(70.1-77.6)$ \\
\hline Sex: Female (\%) & 43 & 44 \\
\hline Median Education (yrs) & $16(14.75-18)$ & $16(14-18)$ \\
\hline Median MMSE score & $29(29-30)$ & $29(29-30)$ \\
\hline Median follow (months) & $30(24-55.5)$ & $30(18-48)$ \\
\hline
\end{tabular}

\section{Images and preprocessing pipeline}

Baseline T1-weighted (T1w) magnetic resonance images (MRIs) were downloaded from ADNI and processed initially through a fully automatic pipeline (Aubert-Broche et al., 2013) that consisted in denoising (Coupe et al., 2008), correction of inhomogeneity using N3 (Sled, Zijdenbos, \& Evans, 1998), registration to pseudo-Talairach stereotaxic space (Collins, Neelin, Peters, \& Evans, 1994) using a population-specific template (Fonov et al., 2011), and brain extraction using BEaST (Eskildsen et al., 2012).

\section{Hippocampal Volumetry}

The hippocampus was segmented automatically using a multi-template patch-based segmentation method (Coupé et al., 2011; A. Zandifar et al., 2017), which uses a training library for MRI volumes with manually traced hippocampi. While the patch-based strategy drastically increases the number of sample library patches involved in label assignment, it reduces susceptibility to registration error. The final label was assigned based on non-local means: each patch was weighted 
based on its similarity to the target patch and the final label (hippocampus or background) was assigned based on a weighted average over all similar patches. The hippocampal volume was estimated by counting voxels in pseudo-Talairach stereotaxic space, thus inherently normalized for head size.

\section{Hippocampal SNIPE Scoring}

A similar approach to the segmentation strategy was followed to assign a grading value to each hippocampi (Coupé, Eskildsen, Manjón, Fonov, \& Collins, 2012). The SNIPE score metric shows how much an image patch is intensity-wise similar to either a cognitively healthy or an AD cohort of training subjects. In fact, instead of hippocampal labels, the diagnosis label of the subjects in the template library is incorporated $(-1$ for $\mathrm{AD},+1$ for $\mathrm{CH})$. The similarity for each voxel is defined based on its corresponding patch. The final score of each voxel is defined based on non-local average over the most similar patches.

The group similarity metric was averaged over the structure area, which is defined by the segmentation process, to assign a value to the whole structure (Coupé, Eskildsen, Manjón, Fonov, \& Collins, 2012). In this study, we computed volumes and SNIPE scores for both left and right hippocampi on our whole cohort using the same training library as the one described in (Coupe et al., 2015). The template library images are all drawn from ADNI-1 dataset. There was no statistically significant difference between the $\mathrm{AD}$ and $\mathrm{CN}$ group in age or gender using a Generalized Linear Model (GLM). Since some of the cognitively healthy subjects are already included in the training library, when computing the SNIPE score for that subject, the corresponding MRI was removed from the training library. 


\section{Statistical analyses and metrics}

All statistical analyses were done using RStudio working under R 3.3.2.

To investigate the sensitivity of each biomarker in detecting between-group differences, we computed the Cohen's d effect size based on both hippocampal volumes and hippocampal SNIPE scores, correcting for age, sex, and ADNI study phase. The correction is done using a similar method to the one presented in (Dukart, Schroeter, Mueller, \& Initiative, 2011) for neurodegeneration and dementia. A linear regression is fitted using only normal controls to regress out the effect of confounding factors such as age while preserving the effect of atrophy-induced neurodegeneration.

The Cohen's d effect size measures the distance between two normal distributions:

$$
\begin{gathered}
\text { Cohen's } d=\frac{\mathrm{m}_{1}-\mathrm{m}_{2}}{\mathrm{SD}_{\text {pooled }}} \\
\mathrm{SD}_{\text {pooled }}=\sqrt{\frac{\mathrm{SD}_{1}{ }^{2}+\mathrm{SD}_{2}{ }^{2}}{2}}
\end{gathered}
$$

where $\mathrm{m}$, and SD are the mean and standard deviation, respectively. Based on a conventional operational definition of Cohen's $d$, small, medium and large effect sizes are defined as $d<0.5$, $0.5<d<0.8$, and $d>0.8$, respectively. We used 200 bootstrapped replicates to obtain a more robust estimation of the effect size.

Further, we used a linear regression model to estimate the association of each marker with age in each clinical group, correcting for sex and ADNI study phase. The linear regression model was fitted using the group label and age as independent variable to and corrected marker values (as dependent variables. In other words, changes in each marker value are modeled using age and the study group of the subject. Therefore, this experiment shows how each marker changes versus age 
in each clinical group. This can be considered as a comparison between normal and abnormal aging.

\section{Results}

\section{Cohen's d Effect size}

The mean effect size (standard deviation) was $0.3415(0.1255)$ for hippocampal volume, and $0.5884(0.1215)$ for hippocampal SNIPE score. A pairwise t-test showed that effect sizes were significantly different between the two markers $\left(p<2.2 e^{-16}\right)$. Hippocampal SNIPE score shows medium effect size, while hippocampal volume has a small effect size.

\section{Linear Regression}

The graphs show hippocampal volumes (Figure 1) and SNIPE scores (Figure 2) plotted against age for both left and right hippocampi. The t-statistics and corresponding p-values show that there is a significant difference between Stables and Progressors clinical groups in either hippocampal volumes or SNIPE scores (hippocampal volumes: $t=2.509(p=0.012), t=2.686(p=$ 0.007; SNIPE scores: $t=3.222(P=0.00135)$ and $t=4.601\left(p=5.3 e^{-6}\right)$, for left and right respectively). There was a significant difference between Stable and Progressor groups in all experimental settings. Figure 2 shows however how this difference is emphasized in hippocampal SNIPE scores, and furthermore, is more dominant in the right hippocampus. 

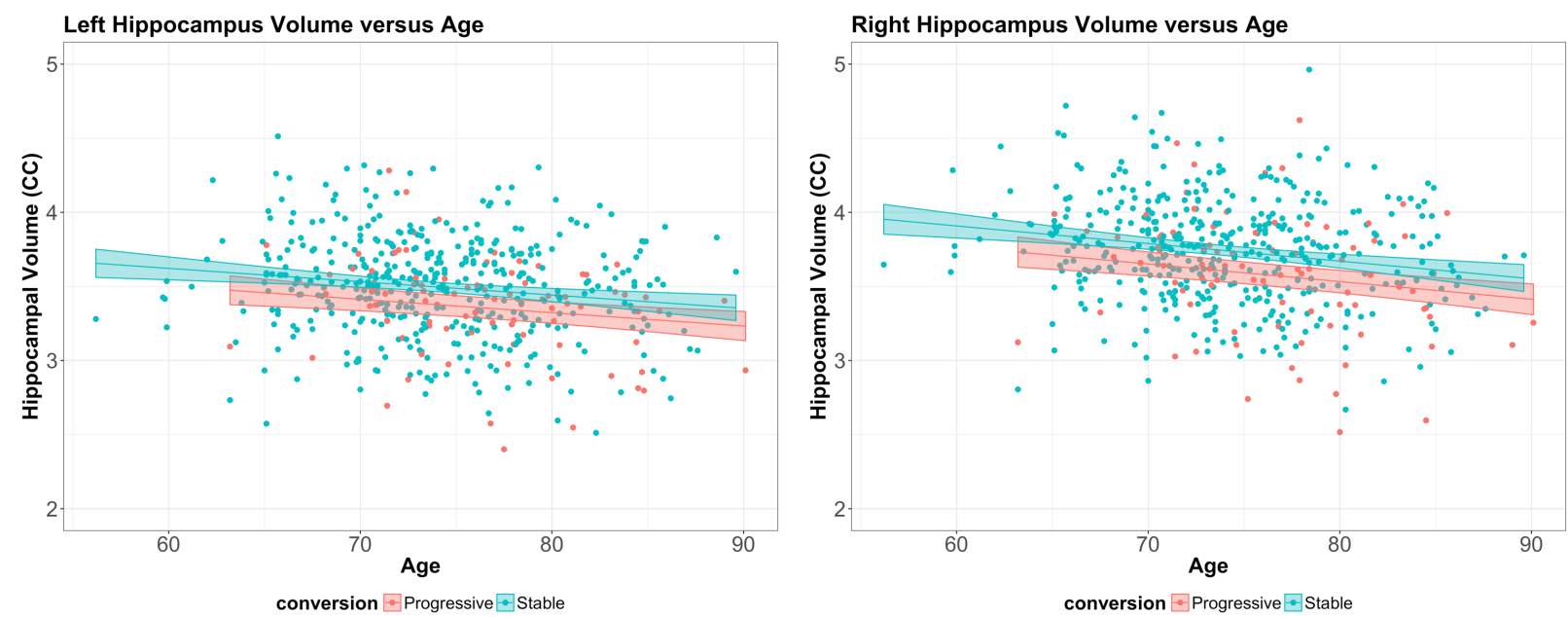

Figure 1 Hippocampal volume versus age for left and right hippocampus. The colors represent different clinical groups. Stable group consists the subjects who remained stable, while progressive group shows the subjects who progressed to $\mathrm{MCl}$ during the follow-up. The reported values are in cubic centimeters
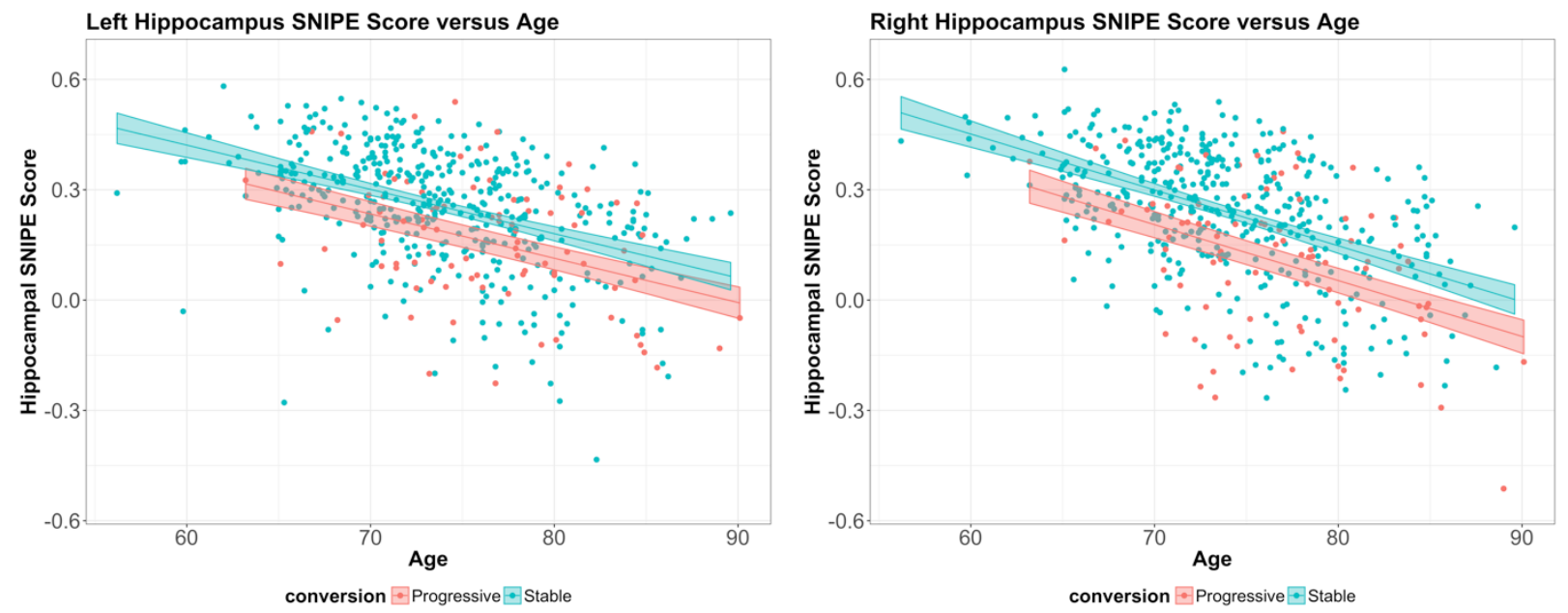

Figure 2 Hippocampal SNIPE score versus age for left and right hippocampus. The colors represent different clinical groups. Stable group consists the subjects who remained stable, while progressive group shows the subjects who progressed to $\mathrm{MCl}$ during the follow-up.

\section{Discussion}

Early detection of Alzheimer's disease pathology in the asymptomatic aging population may increase the effectiveness of interventional procedures to delay dementia onset (Ngandu et al., 2015). In this study we demonstrated that both hippocampi are affected even in pre-clinical stage, when the subject is considered cognitively healthy. These results are in line with the hypothesis 
behind the Jack biomarker model, which shows neurodegenerative atrophy, which can be captured by structural MRI, occurs before clinical symptom appear (Clifford R. Jack et al., 2010). Our effect size analyses show that Cohen's $d$ for between-group differences using hippocampal volume is small during this preclinical stage, especially when compared to the large effect size between AD patients and age-matched $\mathrm{CH}$ subjects (A. Zandifar et al., 2017). This suggests that to better capture early disease impact, there is a need to use more sophisticated feature extraction techniques such as our SNIPE marker, or hippocampal texture (Coupé, Eskildsen, Manjón, Fonov, \& Collins, 2012; Sorensen et al., 2016). In this study hippocampal SNIPE scoring showed medium effect sizes between Stable and Progressor groups, demonstrating a higher sensitive to early AD-related changes than hippocampal volume. This observation is consistent with our previous study in MCI population where a predictive model using hippocampal SNIPE scores led to higher accuracy when compared to using hippocampal volume (Coupé, Eskildsen, Manjón, Fonov, \& Collins, 2012; Coupe et al., 2015).

In order to show how ageing affects the markers in each group, we plotted the markers against age for both Stable and Progressor groups. Our result shows that there is a significant difference between the Stable group and the Progressor considering both markers, and this difference is larger for SNIPE score. However, our data did not demonstrate a different slope over time for each group. This suggests that the disease process may be delayed in the Stable group.

We also noticed that the difference between the groups in the linear regression is larger for the right hippocampus. A two-way ANOVA with hemisphere and marker (hippocampus volume and SNIPE score) as independent variables shows that the difference is significant both between the hemispheres and the markers. However, since previous studies have shown no significant difference for hippocampal hemisphere in AD detection (C. R. Jack et al., 1997), or some even showed the difference is more emphasized on the left side (Shi, Liu, Zhou, Yu, \& Jiang, 2009), we 
believe that the observation could be specific to this particular dataset. Therefore, we decided to use mean hippocampal volume averaged over both hemispheres as a more robust marker to measure the effect size.

This paper shows that SNIPE score can even be used as an informative feature in a model to predict future amnestic MCI and future onset of dementia during the preclinical stage. This marker with more validation will probably be able to provide the clinic with a predictive tool that could have a very long prediction time margin, and clinical trials for cohort enrichment strategies.

\section{Acknowledgements:}

Data collection and sharing for this project was funded by the Alzheimer's Disease Neuroimaging Initiative (ADNI) (National Institutes of Health Grant U01 AG024904) and DOD ADNI (Department of Defense award number W81XWH-12-2-0012). ADNI is funded by the National Institute on Aging, the National Institute of Biomedical Imaging and Bioengineering, and through generous contributions from the following: AbbVie, Alzheimer's Association; Alzheimer's Drug

Discovery Foundation; Araclon Biotech; BioClinica, Inc.; Biogen; Bristol-Myers Squibb Company; CereSpir, Inc.; Cogstate; Eisai Inc.; Elan Pharmaceuticals, Inc.; Eli Lilly and Company; EuroImmun; F. Hoffmann-La Roche Ltd and its affiliated company Genentech, Inc.; Fujirebio; GE Healthcare; IXICO Ltd.; Janssen Alzheimer Immunotherapy Research \& Development, LLC.; Johnson \& Johnson Pharmaceutical Research \& Development LLC.; Lumosity; Lundbeck; Merck \& Co., Inc.; Meso Scale Diagnostics, LLC.; NeuroRx Research; Neurotrack Technologies; Novartis Pharmaceuticals Corporation; Pfizer Inc.; Piramal Imaging; Servier; Takeda Pharmaceutical Company; and Transition Therapeutics. The Canadian Institutes of Health Research is providing funds to support ADNI clinical sites in Canada. Private sector contributions are facilitated by the Foundation for the National Institutes of Health (www.fnih.org). The grantee 
organization is the Northern California Institute for Research and Education, and the study is

coordinated by the Alzheimer's Therapeutic Research Institute at the University of Southern

California. ADNI data are disseminated by the Laboratory for Neuro Imaging at the University of

Southern California.

\section{References:}

Albert, M. S., DeKosky, S. T., Dickson, D., Dubois, B., Feldman, H. H., Fox, N. C., ... Phelps, C. H. (2011). The diagnosis of mild cognitive impairment due to Alzheimer's disease: recommendations from the National Institute on Aging-Alzheimer's Association workgroups on diagnostic guidelines for Alzheimer's disease. Alzheimers Dement, 7(3), 270-279. doi:10.1016/j.jalz.2011.03.008

Alzheimer's Disease Neuroimaging Initiative. (2018). study design - background-rationale. Retrieved from http://adni.loni.usc.edu/study-design/background-rationale/

Ashburner, J., Csernansky, J. G., Davatzikos, C., Fox, N. C., Frisoni, G. B., \& Thompson, P. M. (2003). Computer-assisted imaging to assess brain structure in healthy and diseased brains. Lancet Neurol, 2(2), 79-88.

Aubert-Broche, B., Fonov, V. S., Garcia-Lorenzo, D., Mouiha, A., Guizard, N., Coupe, P., ... Collins, D. L. (2013). A new method for structural volume analysis of longitudinal brain MRI data and its application in studying the growth trajectories of anatomical brain structures in childhood. Neuroimage, 82, 393-402. doi:10.1016/j.neuroimage.2013.05.065

Blennow, K., de Leon, M. J., \& Zetterberg, H. (2006). Alzheimer's disease. Lancet, 368(9533), 387-403.

Braak, H., \& Braak, E. (1991). Neuropathological stageing of Alzheimer-related changes. Acta Neuropathologica, 82(4), 239-259. doi:10.1007/BF00308809

Braak, H., \& Braak, E. (1995). Staging of alzheimer's disease-related neurofibrillary changes. Neurobiology of Aging, 16(3), 271-278. doi:10.1016/0197-4580(95)00021-6

Campion, D., Dumanchin, C., Hannequin, D., Dubois, B., Belliard, S., Puel, M., . . Frebourg, T. (1999). Early-onset autosomal dominant Alzheimer disease: prevalence, genetic heterogeneity, and mutation spectrum. American journal of human genetics, 65(3), 664670. doi:10.1086/302553

Chetelat, G., \& Baron, J. C. (2003). Early diagnosis of Alzheimer's disease: contribution of structural neuroimaging. Neurolmage, 18(2), 525-541.

Collins, D. L., Neelin, P., Peters, T. M., \& Evans, A. C. (1994). Automatic 3D intersubject registration of MR volumetric data in standardized Talairach space. Journal of computer assisted tomography, 18(2), 192-205.

Coupé, P., Eskildsen, S. F., Manjón, J. V., Fonov, V. S., \& Collins, D. L. (2012). Simultaneous segmentation and grading of anatomical structures for patient's classification: 
application to Alzheimer's disease. Neuroimage, 59(4), 3736-3747.

doi:10.1016/j.neuroimage.2011.10.080

Coupé, P., Eskildsen, S. F., Manjón, J. V., Fonov, V. S., Pruessner, J. C., Allard, M., . . Initiative, A. s. D. N. (2012). Scoring by nonlocal image patch estimator for early detection of Alzheimer's disease. Neurolmage: clinical, 1(1), 141-152.

Coupe, P., Fonov, V. S., Bernard, C., Zandifar, A., Eskildsen, S. F., Helmer, C., . . Alzheimer's Disease Neuroimaging, I. (2015). Detection of Alzheimer's disease signature in MR images seven years before conversion to dementia: Toward an early individual prognosis. Hum Brain Mapp, 36(12), 4758-4770. doi:10.1002/hbm.22926

Coupé, P., Manjón, J. V., Fonov, V., Pruessner, J., Robles, M., \& Collins, D. L. (2011). Patch-based segmentation using expert priors: application to hippocampus and ventricle segmentation. Neuroimage, 54(2), 940-954. doi:10.1016/j.neuroimage.2010.09.018

Coupe, P., Yger, P., Prima, S., Hellier, P., Kervrann, C., \& Barillot, C. (2008). An optimized blockwise nonlocal means denoising filter for 3-D magnetic resonance images. IEEE transactions on medical imaging, 27(4), 425-441. doi:10.1109/TMI.2007.906087

Csernansky, J. G., Hamstra, J., Wang, L., McKeel, D., Price, J. L., Gado, M., \& Morris, J. C. (2004). Correlations between antemortem hippocampal volume and postmortem neuropathology in AD subjects. Alzheimer Dis Assoc Disord, 18(4), 190-195.

Dukart, J., Schroeter, M. L., Mueller, K., \& Initiative, A. s. D. N. (2011). Age correction in dementia-matching to a healthy brain. PLoS One, 6(7), e22193.

Duyckaerts, C. (2011). Disentangling Alzheimer's disease. Lancet Neurology, 10(9), 774-775. doi:10.1016/S1474-4422(11)70171-5

Eskildsen, S. F., Coupe, P., Fonov, V., Manjon, J. V., Leung, K. K., Guizard, N., . . Alzheimer's Disease Neuroimaging, I. (2012). BEaST: brain extraction based on nonlocal segmentation technique. Neuroimage, 59(3), 2362-2373. doi:10.1016/j.neuroimage.2011.09.012

Fonov, V., Evans, A. C., Botteron, K., Almli, C. R., McKinstry, R. C., \& Collins, D. L. (2011). Unbiased average age-appropriate atlases for pediatric studies. Neuroimage, 54(1), 313327. doi:10.1016/j.neuroimage.2010.07.033

Fox, N. C., \& Schott, J. M. (2004). Imaging cerebral atrophy: normal ageing to Alzheimer's disease. Lancet, 363(9406), 392-394.

Gomez-Isla, T., Hollister, R., West, H., Mui, S., Growdon, J. H., Petersen, R. C., . . Hyman, B. T. (1997). Neuronal loss correlates with but exceeds neurofibrillary tangles in Alzheimer's disease. Annals of Neurology, 41(1), 17-24. doi:10.1002/ana.410410106

Gomez-Isla, T., Price, J. L., McKeel, D. W., Jr., Morris, J. C., Growdon, J. H., \& Hyman, B. T. (1996). Profound loss of layer II entorhinal cortex neurons occurs in very mild Alzheimer's disease. The Journal of neuroscience : the official journal of the Society for Neuroscience, 16(14), 4491-4500.

Grignon, Y., Duyckaerts, C., Bennecib, M., \& Hauw, J. J. (1998). Cytoarchitectonic alterations in the supramarginal gyrus of late onset Alzheimer's disease. Acta Neuropathologica, 95(4), 395-406.

Hyman, B. T., Phelps, C. H., Beach, T. G., Bigio, E. H., Cairns, N. J., Carrillo, M. C., . . Montine, T. J. (2012). National Institute on Aging-Alzheimer's Association guidelines for the 
neuropathologic assessment of Alzheimer's disease. Alzheimer's \& dementia : the journal of the Alzheimer's Association, 8(1), 1-13. doi:10.1016/j.jalz.2011.10.007

Jack, C. R., Jr., Dickson, D. W., Parisi, J. E., Xu, Y. C., Cha, R. H., O'Brien, P. C., . . Petersen, R. C. (2002). Antemortem MRI findings correlate with hippocampal neuropathology in typical aging and dementia. Neurology, 58(5), 750-757.

Jack, C. R., Jr., Knopman, D. S., Jagust, W. J., Petersen, R. C., Weiner, M. W., Aisen, P. S., . . Trojanowski, J. Q. (2013). Tracking pathophysiological processes in Alzheimer's disease: an updated hypothetical model of dynamic biomarkers. Lancet Neurol, 12(2), 207-216. doi:10.1016/S1474-4422(12)70291-0

Jack, C. R., Knopman, D. S., Jagust, W. J., Petersen, R. C., Weiner, M. W., Aisen, P. S., . . . Trojanowski, J. Q. (2013). Tracking pathophysiological processes in Alzheimer's disease: an updated hypothetical model of dynamic biomarkers. Lancet neurology, 12(2), 207216. doi:10.1016/S1474-4422(12)70291-0

Jack, C. R., Knopman, D. S., Jagust, W. J., Shaw, L. M., Aisen, P. S., Weiner, M. W., . . . Trojanowski, J. Q. (2010). Hypothetical model of dynamic biomarkers of the Alzheimer's pathological cascade. Lancet neurology, 9(1), 119-128. doi:10.1016/S14744422(09)70299-6

Jack, C. R., Petersen, R. C., Xu, Y. C., Waring, S. C., O'Brien, P. C., Tangalos, E. G., ... Kokmen, E. (1997). Medial Temporal Atrophy on MRI in Normal Aging and Very Mild Alzheimer's Disease. Neurology, 49(3), 786-794.

Ngandu, T., Lehtisalo, J., Solomon, A., Levälahti, E., Ahtiluoto, S., Antikainen, R., . . Kivipelto, M. (2015). A 2 year multidomain intervention of diet, exercise, cognitive training, and vascular risk monitoring versus control to prevent cognitive decline in at-risk elderly people (FINGER): a randomised controlled trial. The Lancet, 385(9984), 2255-2263. doi:10.1016/s0140-6736(15)60461-5

Oddo, S., Caccamo, A., Tran, L., Lambert, M. P., Glabe, C. G., Klein, W. L., \& LaFerla, F. M. (2006). Temporal profile of amyloid-beta (Abeta) oligomerization in an in vivo model of Alzheimer disease. A link between Abeta and tau pathology. J Biol Chem, 281(3), 15991604. doi:M507892200 [pii]

10.1074/jbc.M507892200

Shi, F., Liu, B., Zhou, Y., Yu, C., \& Jiang, T. (2009). Hippocampal volume and asymmetry in mild cognitive impairment and Alzheimer's disease: Meta-analyses of MRI studies. Hippocampus, 19(11), 1055-1064.

Sled, J. G., Zijdenbos, A. P., \& Evans, A. C. (1998). A nonparametric method for automatic correction of intensity nonuniformity in MRI data. IEEE transactions on medical imaging, 17(1), 87-97. doi:10.1109/42.668698

Sorensen, L., Igel, C., Liv Hansen, N., Osler, M., Lauritzen, M., Rostrup, E., . . Lifestyle Flagship Study of, A. (2016). Early detection of Alzheimer's disease using MRI hippocampal texture. Hum Brain Mapp, 37(3), 1148-1161. doi:10.1002/hbm.23091

West, M. J., Coleman, P. D., Flood, D. G., \& Troncoso, J. C. (1994). Differences in the pattern of hippocampal neuronal loss in normal ageing and Alzheimer's disease. Lancet, 344(8925), 769-772.

Zandifar, A., Fonov, V., Coupe, P., Pruessner, J., Collins, D. L., \& Alzheimer's Disease Neuroimaging, I. (2017). A comparison of accurate automatic hippocampal 
bioRxiv preprint doi: https://doi.org/10.1101/541854; this version posted February 5,2019 . The copyright holder for this preprint (which was not certified by peer review) is the author/funder, who has granted bioRxiv a license to display the preprint in perpetuity. It is made available under aCC-BY-NC-ND 4.0 International license.

segmentation methods. Neuroimage, 155, 383-393.

doi:10.1016/j.neuroimage.2017.04.018

Zandifar, A., Fonov, V., Coupé, P., Pruessner, J. C., \& Collins, D. L. (2014). A Unified Assessment of Fully Automated Hippocampus Segmentation Methods. Alzheimer's \& Dementia, 10(4), P86. doi:10.1016/j.jalz.2014.05.156 